Review

\title{
Role of p53 Family Proteins in Metformin Anti-Cancer Activities
}

\author{
Yong Yi, Wenhua Zhang, Jianqiao Yi and Zhi-Xiong Xiao ${ }^{凶}$ \\ Yong Yi, Wenhua Zhang, JianqiaoYi and Zhi-Xiong Xiao \\ $\triangle$ Corresponding author: Zhi-Xiong Xiao, Ph.D. Email: jimzx@scu.edu.cn; jxiao@bu.edu; Phone: +86-28-8541-0034; Fax: +86-28-8541-0034. \\ (c) Ivyspring International Publisher. This is an open access article distributed under the terms of the Creative Commons Attribution (CC BY-NC) license \\ (https://creativecommons.org/licenses/by-nc/4.0/). See http://ivyspring.com/terms for full terms and conditions.
}

Received: 2018.10.14; Accepted: 2019.04.23; Published: 2019.05.27

\begin{abstract}
Metformin has been used as therapy for type 2 diabetes for many years. Clinical and basic evidence as indicated that metformin has anti-cancer activities. It has been well-established that metformin activates AMP-activated protein kinase (AMPK), which in turn regulates energy homeostasis. However, the mechanistic aspects of metformin anti-cancer activity remain elusive. p53 family proteins, including p53, p63 and p73, have diverse biological functions, including regulation of cell growth, survival, development, senescence and aging. In this review, we highlight the evidence and mechanisms by which metformin inhibits cancer cell survival and tumor growth. We also aimed to discuss the role of p53 family proteins in metformin-mediated suppression of cancer growth and survival.
\end{abstract}

Key words: metformin, AMPK, p53, p63, p73, cancer

\section{Introduction}

Metformin is widely used to treat type 2 diabetes. Metformin enhances cell sensitivity to insulin[1], inhibits hepatic glucose production and promotes peripheral glucose uptake and, consequently, lowers blood glucose levels[2-4]. In addition, metformin can inhibit glucagon-induced elevation of cyclic adenosine monophosphate (cAMP) to decrease blood glucose[5]. In recent years, accumulating evidence indicates that metformin has biological activities beyond the treatment of diabetes, including anti-aging [6, 7] and anti-obesity effects[8, 9]. In fact, metformin was the first FDA-approved drug in a clinical anti-aging trial[10]. Moreover, numerous clinical and basic studies have implicated metformin in tumor suppression[11]. Currently, the FDA has approved more than 300 clinical trials to investigate the effects of metformin on cancer treatment (www.clinicaltrials.gov).

p53 is a critical tumor suppressor gene frequently mutated in human cancers[12] and is the most studied gene in the human genome[13]. p53 protein contains five distinct domains, including an N-terminal transactivation domain (TAD), a proline-rich domain (PRD), a DNA binding domain (DBD), an oligomeri- zation domain (OD), and a C-terminal regulatory domain (CTD)[14] (Figure 1A). p53 family proteins, p63 and p73, contain either a p53-homologous transactivation domain (TAp63 and TAp73) or lack of this domain $(\triangle \mathrm{Np} 63$ and $\Delta \mathrm{Np} 73)$. Together with alternative splicing variants at $\mathrm{C}$-termini, there are multiple p63 and p73 protein isoforms (Figure 1A). Due to similar structures of DNA binding domains and oligomerization domains, p53 family proteins share a subset of down-stream transcription targets, and they can mutually form oligomers [15]. Indeed, p63 and p73 have biological functions similar to that of p53, including regulation of cell growth, survival, development and senescence[14]. However, p63 and p73 have distinct biological functions. For instance, p63 has a critical role in embryonic development and is a pivotal regulator of cell-cell adhesion maintenance [16]. On the other hand, p73 is important for immunological functions and neurological development [17]. With regard to tumorigenesis, unlike the p53 gene that is frequently mutated in human tumors and cancers, there are rare mutations in the p63 or p73 genes although expression of p63 or p73 are often altered. Interestingly, p63 seems to be involved in 
both tumorigenesis and tumor suppression. On the one hand, p63 is often overexpressed in human squamous cell carcinoma and is critical for cell proliferation and growth[18-21]. On the other hand, down-regulation of p63 plays a pivotal role in cancer metastasis[22-25]. Notably, p63 and p73 are required for p53-dependent apoptosis in response to DNA damage[26] (Figure 1B). Mutant p53 can promote cell invasion via the inhibition of TAp63[27].

Deregulated energetics is a hallmark of cancer cells. Most cancer cells exhibit elevated aerobic glycolysis, known as the Warburg effect[28]. Accumulating evidence reveals that p53 and p53 family members are important in regulation of metabolism. Activation of p53 can transcriptionally inhibit GLUT1 or GLUT4 expression to suppress glucose uptake[29]. In addition, p53 induces expression of TIGAR (TP53-induced glycolysis and apoptosis regulator), a transcription factor involved in suppression of glycolysis and pentose phosphate pathway[30]. Contrary to wild type p53, mutant p53 promotes aerobic glycolysis via increased GLUT1 plasma membrane association[31]. On the other hand, $\Delta \mathrm{Np} 63$ can directly transactivate HK2 expression in facilitating aerobic glycolysis[32]. TAp63 transactivates Sirt1, AMPK $\alpha 2$ or LKB1 in regulation of lipid and glucose metabolism[33]. p73 can also regulate cancer cell metabolism. p73 activates expression of glutaminase-2 (GLS-2) and promotes serine biosynthesis[34]. Metformin has been well established in modulating several master metabolic regulators, such as AMPK, mTOR or HIF1A[35-37]. Therefore, both metformin and p53 family proteins are critically involved in regulation of cancer metabolism, suggesting that function of p53 family proteins and metformin anti-cancer activities are intrinsically connected, which will be our major focus in this review.

\section{Metformin anti-cancer activity in various human cancers}

Metformin has been used to treat type 2 diabetes for many years. Accumulating evidence has indicated that metformin can dramatically reduce tumor incidence in cancer patients with type 2 diabetes[38, 39]. Metformin inhibits growth, survival and metastasis of various cancer cells derived from breast, lung, esophagus, pancreas, liver and blood[40, 41] (Table 1). In addition, it has been recently shown that metformin has anti-tumor activities via an improved immune response[42-44] and modulation of cancerrelated epigenetic modification[45]. The current literature indicates that metformin inhibits tumorigenesis via distinct signaling in different cancer types (Table 1). Importantly, metformin can regulate expression and activities of p53 family proteins in the suppression of tumorigenesis, which are discussed in detail in a later section. Here, we will focus on the effects of metformin on different human cancers.

Breast cancer is the most common cancer in women, especially in 30-59 years old females[46]. It has been reported that long-term use of metformin can dramatically reduce the risk of breast cancer in patients with type 2 diabetes[47]. In addition, breast cancer patients receiving metformin and neoadjuvant chemotherapeutic drugs (Table 1) have a higher pathological complete response rate than patients not receiving metformin[48]. At the molecular levels, metformin can inhibit mTOR signaling or suppress expression of cyclin D1 and ErbB2 (Her2) to inhibit breast cancer cell proliferation and growth[49-51] (Figure 1B). In addition, metformin can selectively inhibit breast cancer stem cells via suppressing nuclear translocation of NF-kB and phosphorylation of STAT3[43, 52] (Figure 1B). Furthermore, recent evidence demonstrated that high expression of peroxisome proliferator-activated receptor gamma coactivator 1-alpha (PGC-1a) can enhance breast cancer resistance to metformin[53]. Currently, there are 42 FDA-approved clinical trials that are investigating the effect of metformin on breast cancer treatment (Table 1).

Table 1. List of selected clinical trials involving in metformin.

\begin{tabular}{|c|c|c|c|}
\hline Cancer Type & Effectors & In Combination with Clinical Drugs & $\begin{array}{l}\text { Clinical Trials } \\
\text { (Approved by FDA) }\end{array}$ \\
\hline Breast Cancer & AMPK/mTOR;NF-кB; STAT3; cyclin D1; Her2 & $\begin{array}{l}\text { 5-FU+Doxorubicin+ Cyclophosphamide; } \\
\text { Doxorubicin+Cyclophosphamide; 5-FU+ } \\
\text { Epirubicin+Cyclophosphamide; Paclitaxel; Docetaxel }\end{array}$ & 42 \\
\hline Lung Cancer & ATM/AMPK; JNK/p38; IL-6 & Afatinib; Crizotinib; Alectinib; Ceritinib; Nivolumab & 15 \\
\hline Esophagus Cancer & $\begin{array}{l}\text { Stat3/Bcl-2; Clycin D1; CDK4; CDK6; AMPK; } \\
\text { NF-кB; AKT }\end{array}$ & 5-FU; Cisplatin & 1 \\
\hline Liver Cancer & $\begin{array}{l}\text { NF-кB; AMPK; AMPK / Foxo3a; ACC; FASN; } \\
\text { ACLY }\end{array}$ & Sorafenib; Rapamycin; Aspirin & 7 \\
\hline Pancreatic Cancer & $\begin{array}{l}\text { Sp transcription factors; AMPK; GPCR; IR; CD44; } \\
\text { EpCAM; EZH2 }\end{array}$ & Rapamycin; Aspirin; Gemcitabine & 16 \\
\hline Leukemia & mTOR; AMPK & Vincristine; Bortezomib; Paclitaxel & 7 \\
\hline
\end{tabular}




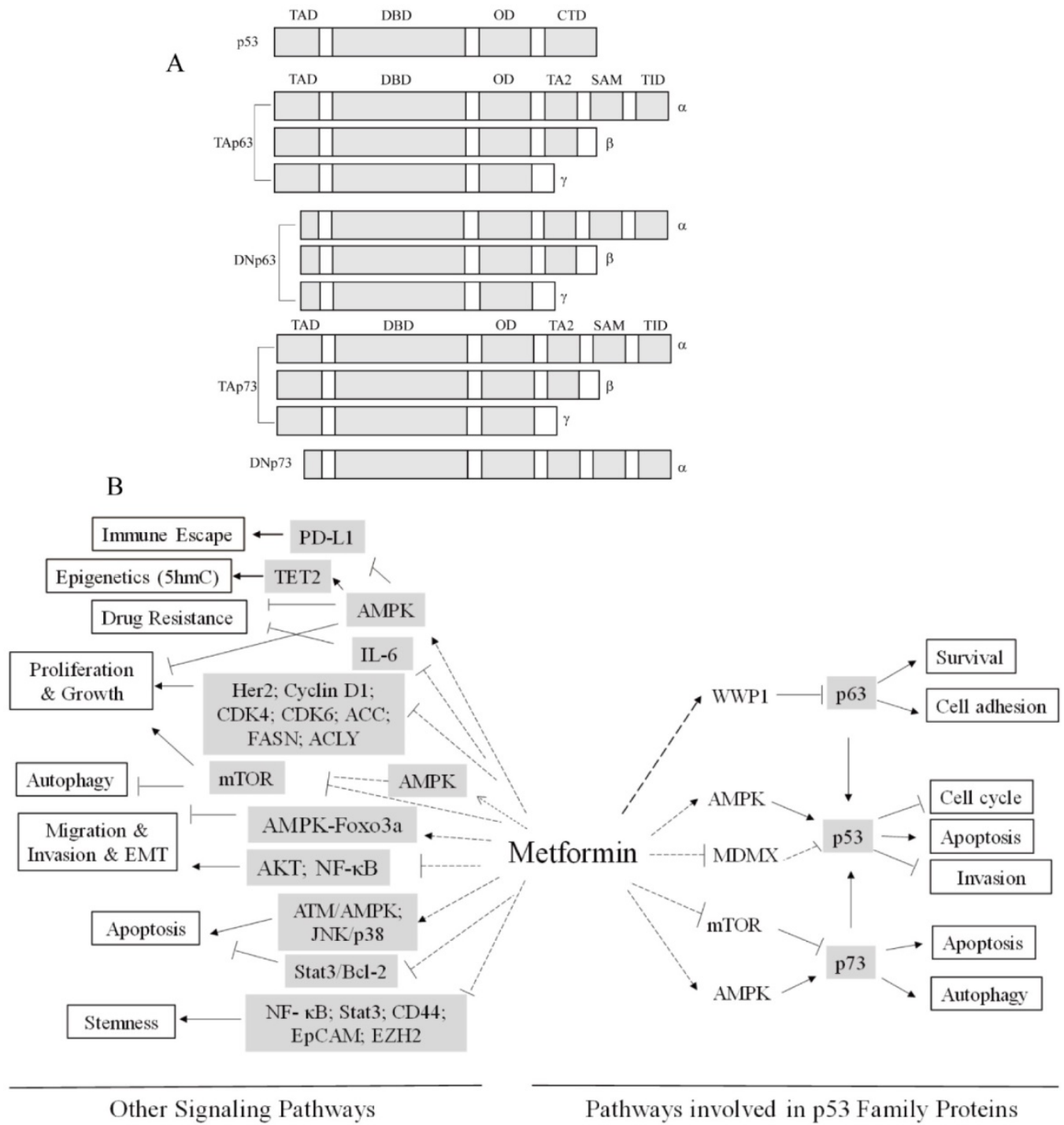

Figure 1. (A) Schematic representation of the structures of $\mathrm{p} 53$, p63 and $\mathrm{p} 73$. Using the alternative transcription starting sites, it generates TA or $\Delta \mathrm{N}$ isoforms. Using the alternative splicing at the C-termini, it generates $\alpha, \beta$ or $\gamma$ isoforms. The transactivation domains (TAD), the DNA-binding domains (DBD), the oligomerization domains (OD), the C-terminal regulatory domains (CTD), the second transactivation domains (TA2), the sterile alpha motif (SAM) and the transactivation inhibitory domains (TID) are indicated. The $\Delta \mathrm{Np} 63$ and $\Delta \mathrm{Np} 73$ variants lack the $\mathrm{N}$-terminal TAD homologues to that of $\mathrm{p} 53$ but their $\mathrm{N}$-termini possess transactivation activities. (B) Pathways Involved in Metformin Anti-Cancer Activities.

Use of metformin is associated with lower risk of lung cancer[54]. Metformin improves outcomes of chemotherapy and/or radiotherapy and survival for non-small cell lung cancer (NSCLC) patients with type 2 diabetes[55]. Metformin can induce apoptosis in lung cancer cells via activating ATM-AMPK and JNK/p38 MAPK pathways[56, 57] (Figure 1B). In addition, metformin can sensitize EGFR-TKI-resistant lung cancer cells through inhibition of IL-6 signaling [58] (Figure 1B). In recent years, several studies have demonstrated that a combination of metformin and other clinically proven drugs are beneficial in lung cancer treatment. For instance, metformin can significantly increase the efficacy of afatinib (EGFR inhibitor) to treat non-small cell lung cancers bearing mutant EGFR[59] (Table 1). In addition, a combination of metformin and ALK inhibitors, including crizotinib, alectinib or ceritinib, exhibits a synthetic effect on $\mathrm{ALK}^{+}$NSCLC cells[60] (Table 1). Most recently, a phase II clinical trial was undertaken to evaluate the effects of a combination of nivolumab (PD-1 monoclonal antibody) and metformin on advanced NSCLC[61] (Table 1). Currently, there are 15 FDA-approved clinical trials to examine metformin anti-lung cancer activity (Table 1). Notably, several clinical studies have shown no clear correlation between metformin use and risk of lung cancer in patients with type 2 diabetes[62, 63]. Thus, more studies are needed to clarify whether metformin is beneficial for lung cancer patients.

Esophageal squamous cell carcinoma (ESCC) is the third most common carcinoma in digestive tracts 
and the sixth leading cause of cancer death in the world[64]. Clinical analyses have indicated that metformin can reduce the risk of esophagus cancer, especially after 2 years of metformin use[65]. Metformin can induce ESCC cell autophagy and apoptosis via inactivation of the Stat3-Bcl-2 pathway[64] (Figure 1B). In addition, metformin can suppress the expression of cyclin D1, CDK4 and CDK6, resulting in ESCC cell growth retardation[66] (Figure 1B). Furthermore, metformin can inhibit migration and invasion of ESCC cells via inhibiting NF-kB nuclear localization[67] or AKT signaling[68] (Figure 1B). Moreover, metformin can enhance esophagus cancer cell sensitivity to chemotherapeutic drugs, 5-FU[69] or cisplatin[70, 71]. Currently, there is one clinical trial approved by the FDA that is exploring the effect of metformin on ESCC (Table 1).

Accumulating evidence has indicated that metformin can inhibit the development of hepatocellular carcinoma (HCC) and improve survival of HCC patients [72-74]. Metformin inhibits NF-kB signaling to suppress HCC cell growth in an AMPK-dependent manner[75]. In addition, metformin inhibits HCC cell migration and down-regulates the epithelial to mesenchymal transition (EMT) via the AMPK-Foxo3a pathway[76] (Figure 1B). Interestingly, metformin inhibits liver tumor growth via suppressing expression of several lipogenic enzymes such as Acetyl-CoA carboxylase (ACC), fatty acid synthase (FASN) and ATP citrate lyase (ACLY), independent of AMPK[77]. Therefore, it seems that metformin inhibits HCC development in both AMPK-dependent and AMPK-independent pathways (Figure 1B). Notably, a combination of metformin and sorafenib, the first-line therapeutic drug for HCC treatment, can significantly inhibit HCC cell proliferation and induce HCC cell autophagy via suppressing mTOR signaling[78] (Figure 1B). However, several clinical studies have shown that chronic treatment with metformin can increase HCC tumor aggressiveness and resistance to sorafenib[79] or have shown no superior efficacy when adding metformin to sorafenib in the treatment of advanced HCC[80]. Furthermore, combined treatment with metformin and rapamycin or aspirin is beneficial to HCC patients[81, 82]. Most recently, it was shown that silencing of hexokinase 2 (HK2) can sensitize HCC cells to metformin[83]. Therefore, a combination of metformin and glycolysis inhibition may be a new strategy for HCC treatment. Currently, there are 7 FDA-approved critical trials that are investigating metformin anti-HCC activity (Table 1 ).

With regard to pancreatic cancers, several clinical studies have indicated that use of metformin is associated with improved outcomes of pancreatic cancer patients with diabetes[84, 85]. Metformin down-regulates expression of $\mathrm{Sp}$ transcription factors, activates AMPK kinase activity, and disrupts crosstalk between G protein-coupled receptors and insulin receptor signaling, leading to inhibition of pancreatic cancer cell proliferation[41, 86, 87]. Metformin can also inhibit stemness of pancreatic cancer stem cells, via re-expression of miRNAs to reduce the expression of cancer stem cell markers, such as CD44, EpCAM and EZH2[88, 89] (Figure 1B). A combination of metformin and rapamycin, aspirin or gemcitabine exhibits better effects in suppressing pancreatic cancer cell proliferation and tumor growth[90-93]. Currently, there are 16 FDA-approved clinical trials that are investigating the effect of metformin on pancreatic cancer (Table 1).

Metformin can inhibit proliferation of T-cell acute lymphoblastic leukemia (T-ALL) cells by inducing autophagy and apoptosis, likely via inhibition of mTOR signaling[94]. Our results showed that metformin can enhance leukemia cell sensitivity to the chemotherapeutic drug, vincristine, via activating AMPK[95] (Figure 1B). In addition, several studies have shown that bortezomib[96] or paclitaxel[97] can enhance metformin anti-leukemia activity. Currently, there are 7 FDA-approved clinical studies that are investigating the effect of metformin on leukemia treatment (Table 1).

Taken together, metformin anti-cancer activities are evident, likely via different mechanisms in various human cancers. Combinational therapy using metformin and other therapeutic drugs may be an effective strategy for cancer treatment.

\section{Metformin, AMPK and p53 family proteins}

It is now clear that metformin can inhibit tumorigenesis in various human cancers and tumors via different signaling pathways. The most demonstrated downstream effector of metformin is AMPK. Different mechanisms by which metformin activates AMPK and the role of AMPK in metformin-mediated biological outcomes have been extensively studied. On the other hand, the p53 family proteins are core components in the regulation of growth and survival upon various stresses. The interplay between metformin, AMPK and p53 family proteins seems critical in metformin-mediated anticancer activities.

AMPK plays a critical role in energy homeostasis via targeting and phosphorylation of mTOR, acetyl-coA carboxylase 1 (ACC1) or modulation of GLUT activity, among others, to regulate protein metabolism, lipid metabolism or glucose metabolism, respectively[98]. Metformin is known to indirectly 
activate AMPK in two different ways. Metformin at a high dose $(\sim \mathrm{mM})$ inhibits the mitochondrial respiratory chain complex I, resulting in an increased AMP/ATP ratio, which in turn promotes AMP binding to AMPK $\gamma$ subunit, AMPK conformational changes and exposure of AMPK $\alpha$ T172 to be phosphorylated and activated by its upstream kinase LKB1 [2]. On the other hand, it was recently shown that metformin at a low dose $(\sim \mu \mathrm{M})$ can activate AMPK through the lysosomal pathway independent of the AMP/ATP ratio[35].

Mounting evidence has indicated that AMPK is involved in cancer cell growth and survival [99]. Clinical studies have demonstrated that AMPK activity is decreased in cancer $[75,95,100]$. Lack of LKB1, a direct AMPK upstream kinase, leads to inhibited AMPK activity[101]. In fact, knockout of LKB1 promotes K-Ras ${ }^{\mathrm{G} 12 \mathrm{D}}$-induced lung cancer metastasis[102]. In addition, activated AKT can directly phosphorylate AMPK $\alpha$ on Serine 485, resulting in AMPK conformational changes and blockage of AMPK $\alpha$ T172 from phosphorylation[103, 104]. Recent studies demonstrated that ubiquitin ligases UBE2O or MAGE-A3/6-TRIM28 can promote ubiquitination of $\mathrm{AMPK} \alpha$, resulting in proteasomedependent degradation and an increase in cell proliferation and tumor growth[105, 106]. Furthermore, knockout of AMPK $\alpha$ can enhance c-Mycinduced lymphoma progression[107]. Notably, single AMPK $\alpha 1$ or AMPK $\alpha 2$ knockout mice do not exhibit accelerated tumor formation[108-110], suggesting that lack of AMPK $\alpha$, alone, is not sufficient in driving tumorigenesis.

Numerous studies have indicated that activation of AMPK inhibits cell proliferation and tumor growth[111]. Glucose deprivation can induce cell death via activation of AMPK[112]. Activation of AMPK can also affect multiple signaling, including mTOR, p53 and NF-kB, to regulate cell growth and survival[113-115]. Currently, small molecules, such as berberine, AICAR and OSU-53[116-118], have been shown to activate AMPK in suppression of cell proliferation and tumor growth, Currently, the role of AMPK in the metformin anti-cancer activities has been extensively studied. Metformin inhibits cell proliferation and tumor growth in AMPK-dependent or AMPK-independent pathways[36, 119]. It has been reported that metformin can reverse multidrug resistance via activating AMPK[120]. Interestingly, several clinical drugs, including paclitaxel and aspirin, can enhance metformin-mediated activation of AMPK to inhibit tumor growth [93, 121]. Most recently, it has been shown that metformin is involved in cancer immune response via activating AMPK, which directly phosphorylates PD-L1 on S195 resulting in ER-associated degradation (ERAD)[44]. In addition, Metformin can also regulate epigenetic modifications by promoting AMPK-mediated phosphorylation of TET2 on S99, resulting in TET2 protein stabilization and accumulation of 5-hydroxymethylcytosine (5hmC) [45].

Activated AMPK can induce phosphorylation of p53 on serine 15, leading to cell-cycle arrest[113]. In addition, AMPK can phosphorylate SIRT1 on Thr344 and inhibit its deacetylase activity toward p53, resulting in enhanced p53 transactivation activity to promote apoptosis of HCC cells[115]. AMPK can also directly phosphorylate p73 on S426, leading to p73 nuclear accumulation, which in turn promotes apoptosis[122]. In contrast, the AMPK catalytic subunit, $\mathrm{AMPK} \alpha$, can interact with $\mathrm{p} 73 \alpha$, promoting p73 $\alpha$ degradation independent of AMPK kinase activity[123]. Hence, the precise effects of AMPK on p73 and its biological outcomes need to be further investigated. With regard to the effects of AMPK on p63, much less is known. Recently, it was reported that lovastatin, a statin drug used for lowering cholesterol, can induce phosphorylation of p63 via activating AMPK-p38MAPK signaling[124].

\section{The role of p53 in metformin anti-cancer action}

Several reports have shown that p53 is involved in metformin anti-cancer action[125-127]. Metformin activates AMPK, which induces p53 phosphorylation and activation to inhibit melanoma invasion and metastasis[125]. Metformin can also inhibit MDMX expression and reduce MDMX-p53 interactions, leading to p53 activation and cell death in lymphoma cells[126] (Figure 1B). A combination of metformin and 2-deoxyglucose induces apoptosis in prostate cancer cells in a p53-dependent manner[127]. Several studies have shown that metformin inhibits the viability of cancer cells lacking p53[128, 129]. Moreover, metformin can sensitize p53-deficient colorectal cancer cells to radiotherapy[129]. Therefore, these results indicate that metformin inhibits cancer cell growth and survival in both p53-dependent and p53-independent ways.

Reactive oxygen species (ROS) play an critical role in regulation of tumorigenesis and cellular response to anticancer therapies[130]. Metformin can reduce cancer risk via inhibiting cellular ROS production[131]. Mechanistically, metformin suppresses ROS production via inhibiting $\mathrm{NAD}(\mathrm{P}) \mathrm{H}$ oxidase activity or activating AMPK/FOXO3a, which facilitates antioxidant thioredoxin[132, 133]. Notably, ROS can act as an upstream factor that activates p53 and a downstream effector of p53 that mediates apoptosis (reference). However, whether ROS-p53 or 
p53-ROS axis plays a role in metformin-mediated anticancer activity need to be further investigated.

\section{Role of p63 in metformin anti-cancer action}

$\Delta \mathrm{Np} 63 \mathrm{a}$ is the predominant $\mathrm{p} 63$ protein isoform expressed in epithelial cells[134]. $\Delta \mathrm{Np} 63 \alpha$ is a critical transcriptional regulator of the cell adhesion program, through regulation of a subset of adhesion molecules, including integrin $\beta 4$, integrin $\alpha 6$ and fibronectin $1[16] . \Delta N p 63 a$ expression is significantly reduced in advanced human cancers, including breast cancer, lung cancer and bladder cancer[22, 135, 136]. Work from our lab and others showed that oncogenic PI3K/HER2/Ras commonly inhibits expression of $\triangle \mathrm{Np63a}$, facilitating cell migration and cancer metastasis[22, 25]. Furthermore, we found that MKP3 and CD82 are the downstream targets of $\triangle N p 63 a$ in regulating cancer metastasis[23, 24].

Notably, $\Delta \mathrm{Np} 63 \mathrm{a}$ has been shown to be overexpressed in squamous cell carcinoma (SCC) and to promote SCC cell proliferation and survival[21]. Our study indicated that protein isomerase Pin1 directly binds to and stabilizes $\Delta \mathrm{Np} 63 \mathrm{a}$ to promote proliferation of head and neck squamous cell carcinoma cells (HNSCC)[19]. $\triangle \mathrm{Np63a}$ can also suppress p73-induced apoptosis to promote survival of HNSCC cells[21]. Our recent study demonstrated that metformin dramatically inhibits $\triangle N p 63 a$ protein expression to inhibit SCC cell viability under glucose starvation or in combination with 2-deoxyglucose (2-DG)[18]. At the molecular level, metformin upregulates WWP1 expression to destabilize $\triangle \mathrm{Np} 63 \mathrm{a}$ protein independent of AMPK[18], indicating that $\triangle \mathrm{Np} 63 \mathrm{a}$ is an important downstream target of metformin (Figure 1B).

\section{Role of p73 in metformin anti-cancer activity}

Like p53, p73 has anti-proliferative and proapoptotic activities[137]. p73 plays a critical role in cancer cell response to chemotherapy[138]. Unlike p53, the p73 gene is rarely mutated in human tumors. Metformin can stabilize p73 via activation of AMPK to induce cell apoptosis[122] (Figure 1B). Metformin can also increase p73 protein expression to promote cell autophagy[139] (Figure 1B). These results indicate that p73 is important in metformin anti-cancer action. Further in vivo studies are needed to verify whether metformin targets p73 in the regulation of cancer cell growth and survival.

\section{Conclusion}

There is no doubt that metformin possesses anti-cancer activities. The effects of metformin in suppression of tumorigenesis have been demonstrated in many human cancers derived from breast, lung, esophagus, liver, pancreas and blood. In the past decade, many signaling pathways impacted by metformin have been identified. AMPK is the most studied down-stream effector of metformin. Metformin can activate AMPK in mitochondriadependent and lysosome-dependent pathways. Activated AMPK inhibits tumor growth and survival via regulating its downstream targets including p53/p63/p73, mTOR, ACC, and MAPK. p53 family proteins are intimately involved in metforminmediated inhibition of cell growth and survival. In particular, p63 emerges as a novel metformin target in the inhibition of cell survival and tumor growth. However, whether p63 is a critical target of metformin in cancer patients needs to be further investigated.

Metformin is speculated to be a promising candidate drug for cancer treatment in combination with therapeutic drugs. Currently, more than 300 FDA-approved clinical trials are undergoing investigation of metformin anti-cancer activity, including numerous studies that use a combination of metformin with other drugs such as afatinib [59], sorafenib [79] and chemotherapeutic drugs[48, 140]. It would be interesting to examine a combination of metformin with p53/p63/p73-targeted drugs, if available, in cancer treatment.

\section{Acknowledgments}

This work was supported by the China Postdoctoral Science Foundation (2018M631081) and post-doctoral grant of Sichuan University (2018SCU12 054) to Y.Y. and National Natural Science Foundation of China (81802951) to YY and (81330054 and 81520108020) to Z.X.X.

\section{Author Contributions}

$Y Y$ and $X Z X$ idealized the general structure of the text, YY, ZWH and XZX wrote the paper, idealized and designed the figures.

\section{Competing Interests}

The authors have declared that no competing interest exists.

\section{References}

1. Verma S, Yao L, Dumont AS, et al. Metformin treatment corrects vascular insulin resistance in hypertension. Journal of hypertension. 2000; 18: 1445-50.

2. Owen MR, Doran E, Halestrap AP. Evidence that metformin exerts its anti-diabetic effects through inhibition of complex 1 of the mitochondrial respiratory chain. Biochem J. 2000; 348 Pt 3: 607-14.

3. Musi N, Hirshman MF, Nygren J, et al. Metformin increases AMP-activated protein kinase activity in skeletal muscle of subjects with type 2 diabetes. Diabetes. 2002; 51: 2074-81.

4. Zhou G, Myers R, Li Y, et al. Role of AMP-activated protein kinase in mechanism of metformin action. The Journal of clinical investigation. 2001; 108: $1167-74$. 
5. Miller RA, Chu Q, Xie J, et al. Biguanides suppress hepatic glucagon signalling by decreasing production of cyclic AMP. Nature. 2013; 494: 256-60.

6. Cabreiro F, Au C, Leung KY, et al. Metformin retards aging in C. elegans by altering microbial folate and methionine metabolism. Cell. 2013; 153: 228-39.

7. Barzilai N, Crandall JP, Kritchevsky SB, et al. Metformin as a Tool to Target Aging. Cell Metab. 2016; 23: 1060-5.

8. Seifarth C, Schehler B, Schneider HJ. Effectiveness of metformin on weight loss in non-diabetic individuals with obesity. Exp Clin Endocrinol Diabetes. 2013; 121: $27-31$

9. Desilets AR, Dhakal-Karki S, Dunican KC. Role of metformin for weight management in patients without type 2 diabetes. Ann Pharmacother. 2008; 42: 817-26.

10. Hall SS. A Trial for the ages. Science. 2015; 349: 1274-8.

11. Pernicova I, Korbonits M. Metformin--mode of action and clinical implications for diabetes and cancer. Nat Rev Endocrinol. 2014; 10: 143-56.

12. Hollstein M, Sidransky D, Vogelstein B, et al. p53 mutations in human cancers. Science. 1991; 253: 49-53.

13. Dolgin E. The most popular genes in the human genome. Nature. 2017; 551: 427-31.

14. Bergholz J, Xiao ZX. Role of p63 in Development, Tumorigenesis and Cancer Progression. Cancer Microenviron. 2012; 5: 311-22.

15. Dotsch V, Bernassola F, Coutandin D, et al. p63 and p73, the ancestors of p53. Cold Spring Harb Perspect Biol. 2010; 2: a004887.

16. Carroll DK, Carroll JS, Leong CO, et al. p63 regulates an adhesion programme and cell survival in epithelial cells. Nat Cell Biol. 2006; 8: 551-61.

17. Yang A, Walker N, Bronson R, et al. p73-deficient mice have neurological, pheromonal and inflammatory defects but lack spontaneous tumours. Nature. 2000; 404: 99-103.

18. Yi Y, Chen D, Ao J, et al. Metformin Promotes AMP-activated Protein Kinase-independent Suppression of DeltaNp63alpha Protein Expression and Inhibits Cancer Cell Viability. J Biol Chem. 2017; 292: 5253-61.

19. Li C, Chang DL, Yang Z, et al. Pin1 modulates p63alpha protein stability in regulation of cell survival, proliferation and tumor formation. Cell death \& disease. 2013; 4: e943.

20. Han $\mathrm{A}, \mathrm{Li}$ J, Li $\mathrm{Y}$, et al. p63alpha modulates c-Myc activity via direct interaction and regulation of MM1 protein stability. Oncotarget. 2016; 7: 44277-87.

21. Rocco JW, Leong CO, Kuperwasser $\mathrm{N}$, et al. p63 mediates survival in squamous cell carcinoma by suppression of p73-dependent apoptosis. Cancer Cell. 2006; 9: 45-56

22. $\mathrm{Hu} \mathrm{L}$, Liang $\mathrm{S}$, Chen $\mathrm{H}$, et al. DeltaNp63alpha is a common inhibitory target in oncogenic PI3K/Ras/Her2-induced cell motility and tumor metastasis. Proceedings of the National Academy of Sciences of the United States of America. 2017; 114: E3964-E73.

23. Wu J, Liang S, Bergholz J, et al. DeltaNp63alpha activates CD82 metastasis suppressor to inhibit cancer cell invasion. Cell death \& disease. 2014; 5: e1280.

24. Bergholz J, Zhang Y, Wu J, et al. DeltaNp63alpha regulates Erk signaling via MKP3 to inhibit cancer metastasis. Oncogene. 2014; 33: 212-24

25. Yoh KE, Regunath K, Guzman A, et al. Repression of p63 and induction of EMT by mutant Ras in mammary epithelial cells. Proceedings of the National Academy of Sciences of the United States of America. 2016; 113: E6107-E16.

26. Flores ER, Tsai KY, Crowley D, et al. p63 and p73 are required for p53-dependent apoptosis in response to DNA damage. Nature. 2002; 416: 560-4.

27. Muller PA, Caswell PT, Doyle B, et al. Mutant p53 drives invasion by promoting integrin recycling. Cell. 2009; 139: 1327-41.

28. Hanahan D, Weinberg RA. Hallmarks of cancer: the next generation. Cell. 2011; 144: 646-74.

29. Schwartzenberg-Bar-Yoseph F, Armoni M, Karnieli E. The tumor suppressor p53 down-regulates glucose transporters GLUT1 and GLUT4 gene expression. Cancer research. 2004; 64: 2627-33.

30. Bensaad K, Tsuruta A, Selak MA, et al. TIGAR, a p53-inducible regulator of glycolysis and apoptosis. Cell. 2006; 126: 107-20.

31. Zhang C, Liu J, Liang $Y$, et al. Tumour-associated mutant p53 drives the Warburg effect. Nat Commun. 2013; 4: 2935.

32. Viticchie G, Agostini M, Lena AM, et al. p63 supports aerobic respiration through hexokinase II. Proceedings of the National Academy of Sciences of the United States of America. 2015; 112: 11577-82.

33. Su X, Gi YJ, Chakravarti D, et al. TAp63 is a master transcriptional regulator of lipid and glucose metabolism. Cell Metab. 2012; 16: 511-25.

34. Amelio I, Markert EK, Rufini A, et al. p73 regulates serine biosynthesis in cancer. Oncogene. 2014; 33: 5039-46.

35. Zhang CS, Li M, Ma T, et al. Metformin Activates AMPK through the Lysosomal Pathway. Cell Metab. 2016; 24: 521-2.

36. Ben Sahra I, Regazzetti C, Robert G, et al. Metformin, independent of AMPK, induces mTOR inhibition and cell-cycle arrest through REDD1. Cancer research. 2011; 71: 4366-72.

37. Sheng B, Liu J, Li GH. Metformin preconditioning protects Daphnia pulex from lethal hypoxic insult involving AMPK, HIF and mTOR signaling. Comparative biochemistry and physiology Part B, Biochemistry \& molecular biology. 2012; 163: 51-8.

38. Libby G, Donnelly LA, Donnan PT, et al. New users of metformin are at low risk of incident cancer: a cohort study among people with type 2 diabetes. Diabetes Care. 2009; 32: 1620-5
39. Evans JM, Donnelly LA, Emslie-Smith AM, et al. Metformin and reduced risk of cancer in diabetic patients. BMJ. 2005; 330: 1304-5.

40. Menendez JA, Cufi S, Oliveras-Ferraros C, et al. Metformin and the ATM DNA damage response (DDR): accelerating the onset of stress-induced senescence to boost protection against cancer. Aging. 2011; 3: 1063-77.

41. Kisfalvi K, Eibl G, Sinnett-Smith J, et al. Metformin disrupts crosstalk between $\mathrm{G}$ protein-coupled receptor and insulin receptor signaling systems and inhibits pancreatic cancer growth. Cancer research. 2009; 69: 6539-45.

42. Eikawa S, Nishida M, Mizukami S, et al. Immune-mediated antitumor effect by type 2 diabetes drug, metformin. Proceedings of the National Academy of Sciences of the United States of America. 2015; 112: 1809-14.

43. Hirsch HA, Iliopoulos D, Struhl K. Metformin inhibits the inflammatory response associated with cellular transformation and cancer stem cell growth. Proceedings of the National Academy of Sciences of the United States of America. 2013; 110: 972-7.

44. Cha JH, Yang WH, Xia W, et al. Metformin Promotes Antitumor Immunity via Endoplasmic-Reticulum-Associated Degradation of PD-L1. Mol Cell. 2018; 71: 606-20 e7.

45. Wu D, Hu D, Chen H, et al. Glucose-regulated phosphorylation of TET2 by AMPK reveals a pathway linking diabetes to cancer. Nature. 2018; 559: 637-41.

46. Chen W, Zheng R, Baade PD, et al. Cancer statistics in China, 2015. CA Cancer J Clin. 2016; 66: 115-32.

47. Bodmer M, Meier C, Krahenbuhl S, et al. Long-term metformin use is associated with decreased risk of breast cancer. Diabetes Care. 2010; 33: 1304-8.

48. Jiralerspong S, Palla SL, Giordano SH, et al. Metformin and pathologic complete responses to neoadjuvant chemotherapy in diabetic patients with breast cancer. J Clin Oncol. 2009; 27: 3297-302.

49. Zakikhani M, Dowling $R$, Fantus IG, et al. Metformin is an AMP kinase-dependent growth inhibitor for breast cancer cells. Cancer research. 2006; 66: 10269-73.

50. Vazquez-Martin A, Oliveras-Ferraros C, Menendez JA. The antidiabetic drug metformin suppresses HER2 (erbB-2) oncoprotein overexpression via inhibition of the mTOR effector p70S6K1 in human breast carcinoma cells. Cell Cycle. 2009; 8: 88-96.

51. Ben Sahra I, Laurent K, Loubat A, et al. The antidiabetic drug metformin exerts an antitumoral effect in vitro and in vivo through a decrease of cyclin D1 level. Oncogene. 2008; 27: 3576-86.

52. Hirsch HA, Iliopoulos D, Tsichlis PN, et al. Metformin selectively targets cancer stem cells, and acts together with chemotherapy to block tumor growth and prolong remission. Cancer research. 2009; 69: 7507-11.

53. Andrzejewski S, Klimcakova E, Johnson RM, et al. PGC-1alpha Promotes Breast Cancer Metastasis and Confers Bioenergetic Flexibility against Metabolic Drugs. Cell Metab. 2017; 26: 778-87 e5.

54. Zhang ZJ, Bi Y, Li S, et al. Reduced risk of lung cancer with metformin therapy in diabetic patients: a systematic review and meta-analysis. Am J Epidemiol. 2014; 180: 11-4

55. Tan BX, Yao WX, Ge J, et al. Prognostic influence of metformin as first-line chemotherapy for advanced nonsmall cell lung cancer in patients with type 2 diabetes. Cancer. 2011; 117: 5103-11.

56. Storozhuk Y, Hopmans SN, Sanli T, et al. Metformin inhibits growth and enhances radiation response of non-small cell lung cancer (NSCLC) through ATM and AMPK. Br J Cancer. 2013; 108: 2021-32.

57. Wu N, Gu C, Gu H, et al. Metformin induces apoptosis of lung cancer cells through activating JNK/p38 MAPK pathway and GADD153. Neoplasma. 2011; 58: 482-90.

58. Li L, Han R, Xiao H, et al. Metformin sensitizes EGFR-TKI-resistant human lung cancer cells in vitro and in vivo through inhibition of IL-6 signaling and EMT reversal. Clin Cancer Res. 2014; 20: 2714-26.

59. Pedro Barrios-Bernal NYH-P, Giovanny Soca-Chafre, Mario Orozco-Morales and Oscar Arrieta. Abstract 3554: The combined effect of afatinib and metformin on glycolytic regulation in EGFR-mutant non-small cell lung cancer. Cancer research. 2017.

60. Mi Young Kim GOK, Dong Hoon Shin, Tae Min Kim and Jin-Soo Kim. Abstract 759: Metformin enhances the benefit of ALK inhibitors in ALK translocation-positive non-small cell lung cancer cells. Cancer research. 2015.

61. Y. Chae WI, S. Pai, R. Costa, T. Taxter, N. Mohindra, V. Villaflor, B. Pro, F. Giles. P1.04-005 Phase 2 Study of Nivolumab and Metformin in Advanced Non-Small Cell Lung Cancer with and without Prior Treatment with PD-1/PD-L1 Inhibitors. Journal of Thoracic Oncology. 2017; 12: S1974.

62. Smiechowski BB, Azoulay L, Yin H, et al. The use of metformin and the incidence of lung cancer in patients with type 2 diabetes. Diabetes Care. 2013; 36: 124-9.

63. Bodmer M, Becker C, Jick SS, et al. Metformin does not alter the risk of lung cancer: a case-control analysis. Lung Cancer. 2012; 78: 133-7.

64. Feng Y, Ke C, Tang Q, et al. Metformin promotes autophagy and apoptosis in esophageal squamous cell carcinoma by downregulating Stat3 signaling. Cell death \& disease. 2014; 5: e1088.

65. Tseng $\mathrm{CH}$. Metformin and esophageal cancer risk in Taiwanese patients with type 2 diabetes mellitus. Oncotarget. 2017; 8: 18802-10.

66. Kobayashi M, Kato $\mathrm{K}$, Iwama $\mathrm{H}$, et al. Antitumor effect of metformin in esophageal cancer: in vitro study. Int J Oncol. 2013; 42: 517-24.

67. Sekino N, Kano M, Matsumoto Y, et al. Antitumor effects of metformin are a result of inhibiting nuclear factor kappa $\mathrm{B}$ nuclear translocation in esophageal squamous cell carcinoma. Cancer Sci. 2018; 109: 1066-74. 
68. He Y, Tan X, Hu H, et al. Metformin inhibits the migration and invasion of esophageal squamous cell carcinoma cells by downregulating the protein kinase B signaling pathway. Oncol Lett. 2018; 15: 2939-45.

69. Honjo S, Ajani JA, Scott AW, et al. Metformin sensitizes chemotherapy by targeting cancer stem cells and the mTOR pathway in esophageal cancer. Int J Oncol. 2014; 45: 567-74.

70. Li PD, Liu Z, Cheng TT, et al. Redox-dependent modulation of metformin contributes to enhanced sensitivity of esophageal squamous cell carcinoma to cisplatin. Oncotarget. 2017; 8: 62057-68.

71. Wang F, Ding X, Wang T, et al. Metformin inhibited esophageal squamous cell carcinoma proliferation in vitro and in vivo and enhanced the anti-cancer effect of cisplatin. PloS one. 2017; 12: e0174276.

72. Donadon V, Balbi M, Mas MD, et al. Metformin and reduced risk of hepatocellular carcinoma in diabetic patients with chronic liver disease. Liver Int. 2010; 30: 750-8.

73. Ma SJ, Zheng YX, Zhou PC, et al. Metformin use improves survival of diabetic liver cancer patients: systematic review and meta-analysis. Oncotarget. 2016; 7: 66202-11.

74. Lee MS, Hsu CC, Wahlqvist ML, et al. Type 2 diabetes increases and metformin reduces total, colorectal, liver and pancreatic cancer incidences in Taiwanese: a representative population prospective cohort study of 800,000 individuals. BMC Cancer. 2011; 11: 20.

75. Zheng L, Yang W, Wu F, et al. Prognostic significance of AMPK activation and therapeutic effects of metformin in hepatocellular carcinoma. Clin Cancer Res. 2013; 19: 5372-80.

76. S. Di Matteo AL, D. Costantini, L. Nevi, E. Manzi, C. Napoletano, J. Faccioli, F. Giulitti, A.M. DeRose, M.C. Bragazzi, G. Grazi, P.B. Berloco, F. Giuliante, V. Cardinale, G. Carpino, D. Alvaro. Metformin reduces cell migration and down-regulates epithelial to mesenchymal transition by AMPK / Foxo3a pathway in human intrahepatic cholangiocarcinoma. Journal of Hepatology. 2017; 66: Page S636

77. Bhalla K, Hwang BJ, Dewi RE, et al. Metformin prevents liver tumorigenesis by inhibiting pathways driving hepatic lipogenesis. Cancer Prev Res (Phila). 2012; 5: 544-52.

78. Ling S, Song L, Fan N, et al. Combination of metformin and sorafenib suppresses proliferation and induces autophagy of hepatocellular carcinoma via targeting the mTOR pathway. Int J Oncol. 2017; 50: 297-309.

79. Casadei Gardini A, Faloppi L, De Matteis S, et al. Metformin and insulin impact on clinical outcome in patients with advanced hepatocellular carcinoma receiving sorafenib: Validation study and biological rationale. Eur J Cancer. 2017; 86: 106-14.

80. Saber SESRHFALHAHEOHMMHRKES. Prognostic significance of VEGF and HIF 1 ? in hepatocellular carcinoma patients receiving sorafenib versus metformin sorafenib combination Annals of Oncology. 2017; 28

81. Tsai HH, Lai HY, Chen YC, et al. Metformin promotes apoptosis in hepatocellular carcinoma through the CEBPD-induced autophagy pathway. Oncotarget. 2017; 8: 13832-45.

82. Abdelmonsif DA, Sultan AS, El-Hadidy WF, et al. Targeting AMPK, mTOR and beta-Catenin by Combined Metformin and Aspirin Therapy in HCC: An Appraisal in Egyptian HCC Patients. Mol Diagn Ther. 2018; 22: 115-27.

83. DeWaal D, Nogueira V, Terry AR, et al. Hexokinase-2 depletion inhibits glycolysis and induces oxidative phosphorylation in hepatocellular carcinoma and sensitizes to metformin. Nat Commun. 2018; 9: 446.

84. Sadeghi N, Abbruzzese JL, Yeung SC, et al. Metformin use is associated with better survival of diabetic patients with pancreatic cancer. Clin Cancer Res. 2012; 18: 2905-12.

85. Li D, Yeung SC, Hassan MM, et al. Antidiabetic therapies affect risk of pancreatic cancer. Gastroenterology. 2009; 137: 482-8.

86. Nair V, Pathi S, Jutooru I, et al. Metformin inhibits pancreatic cancer cell and tumor growth and downregulates Sp transcription factors. Carcinogenesis. 2013; 34: 2870-9.

87. Karnevi E, Said K, Andersson R, et al. Metformin-mediated growth inhibition involves suppression of the IGF-I receptor signalling pathway in human pancreatic cancer cells. BMC Cancer. 2013; 13: 235.

88. Bao B, Wang $\mathrm{Z}$, Ali S, et al. Metformin inhibits cell proliferation, migration and invasion by attenuating CSC function mediated by deregulating miRNAs in pancreatic cancer cells. Cancer Prev Res (Phila). 2012; 5: 355-64.

89. Lonardo E, Cioffi M, Sancho P, et al. Metformin targets the metabolic achilles heel of human pancreatic cancer stem cells. PloS one. 2013; 8: e76518.

90. Cifarelli V, Lashinger LM, Devlin KL, et al. Metformin and Rapamycin Reduce Pancreatic Cancer Growth in Obese Prediabetic Mice by Distinct MicroRNA-Regulated Mechanisms. Diabetes. 2015; 64: 1632-42.

91. Yue $W$, Zheng $X$, Lin $Y$, et al. Metformin combined with aspirin significantly inhibit pancreatic cancer cell growth in vitro and in vivo by suppressing anti-apoptotic proteins Mcl-1 and Bcl-2. Oncotarget. 2015; 6: 21208-24.

92. Chai $X, C h u H$, Yang $X$, et al. Metformin Increases Sensitivity of Pancreatic Cancer Cells to Gemcitabine by Reducing CD133+ Cell Populations and Suppressing ERK/P70S6K Signaling. Sci Rep. 2015; 5: 14404.

93. Yue W, Yang CS, DiPaola RS, et al. Repurposing of metformin and aspirin by targeting AMPK-mTOR and inflammation for pancreatic cancer prevention and treatment. Cancer Prev Res (Phila). 2014; 7: 388-97.

94. Grimaldi C, Chiarini F, Tabellini G, et al. AMP-dependent kinase/mammalian target of rapamycin complex 1 signaling in T-cell acute lymphoblastic leukemia: therapeutic implications. Leukemia. 2012; 26: 91-100.
95. Yi Y, Gao L, Wu M, et al. Metformin Sensitizes Leukemia Cells to Vincristine via Activation of AMP-activated Protein Kinase. J Cancer. 2017; 8: 2636-42.

96. Jagannathan S, Abdel-Malek MA, Malek E, et al. Pharmacologic screens reveal metformin that suppresses GRP78-dependent autophagy to enhance the anti-myeloma effect of bortezomib. Leukemia. 2015; 29: 2184-91.

97. Asik A, Kayabasi C, Ozmen Yelken B, et al. Antileukemic effect of paclitaxel in combination with metformin in HL-60 cell line. Gene. 2018; 647: 213-20.

98. Hardie DG, Ross FA, Hawley SA. AMPK: a nutrient and energy sensor that maintains energy homeostasis. Nat Rev Mol Cell Biol. 2012; 13: 251-62.

99. Luo Z, Saha AK, Xiang X, et al. AMPK, the metabolic syndrome and cancer. Trends Pharmacol Sci. 2005; 26: 69-76.

100. Hadad SM, Baker L, Quinlan PR, et al. Histological evaluation of AMPK signalling in primary breast cancer. BMC Cancer. 2009; 9: 307

101. Carretero J, Medina PP, Blanco R, et al. Dysfunctional AMPK activity, signalling through mTOR and survival in response to energetic stress in LKB1-deficient lung cancer. Oncogene. 2007; 26: 1616-25.

102. Ji H, Ramsey MR, Hayes DN, et al. LKB1 modulates lung cancer differentiation and metastasis. Nature. 2007; 448: 807-10.

103. Ning J, Xi G, Clemmons DR. Suppression of AMPK activation via S485 phosphorylation by IGF-I during hyperglycemia is mediated by AKT activation in vascular smooth muscle cells. Endocrinology. 2011; 152: 3143-54.

104. Hawley SA, Ross FA, Gowans GJ, et al. Phosphorylation by Akt within the ST loop of AMPK-alpha1 down-regulates its activation in tumour cells. Biochem J. 2014; 459: 275-87.

105. Pineda CT, Ramanathan S, Fon Tacer K, et al. Degradation of AMPK by a cancer-specific ubiquitin ligase. Cell. 2015; 160: 715-28.

106. Hardie DG. An Oncogenic Role for the Ubiquitin Ligase UBE2O by Targeting AMPK-alpha2 for Degradation. Cancer Cell. 2017; 31: 163-5.

107. Faubert B, Boily G, Izreig S, et al. AMPK is a negative regulator of the Warburg effect and suppresses tumor growth in vivo. Cell Metab. 2013; 17: 113-24.

108. Narkar VA, Downes M, Yu RT, et al. AMPK and PPARdelta agonists are exercise mimetics. Cell. 2008; 134: 405-15.

109. Viollet B, Andreelli F, Jorgensen SB, et al. The AMP-activated protein kinase alpha2 catalytic subunit controls whole-body insulin sensitivity. J Clin Invest. 2003; 111: 91-8.

110. Jorgensen SB, Wojtaszewski JF, Viollet B, et al. Effects of alpha-AMPK knockout on exercise-induced gene activation in mouse skeletal muscle. FASEB J. 2005; 19: 1146-8.

111. Motoshima $\mathrm{H}$, Goldstein BJ, Igata $\mathrm{M}$, et al. AMPK and cell proliferation--AMPK as a therapeutic target for atherosclerosis and cancer. J Physiol. 2006; 574: 63-71.

112. Priebe A, Tan L, Wahl H, et al. Glucose deprivation activates AMPK and induces cell death through modulation of Akt in ovarian cancer cells. Gynecologic oncology. 2011; 122: 389-95.

113. Jones RG, Plas DR, Kubek $S$, et al. AMP-activated protein kinase induces a p53-dependent metabolic checkpoint. Mol Cell. 2005; 18: 283-93.

114. Shi WY, Xiao D, Wang L, et al. Therapeutic metformin/AMPK activation blocked lymphoma cell growth via inhibition of mTOR pathway and induction of autophagy. Cell death \& disease. 2012; 3: e275.

115. Lee CW, Wong LL, Tse EY, et al. AMPK promotes p53 acetylation via phosphorylation and inactivation of SIRT1 in liver cancer cells. Cancer research. 2012; 72: 4394-404.

116. Park JJ, Seo SM, Kim EJ, et al. Berberine inhibits human colon cancer cell migration via AMP-activated protein kinase-mediated downregulation of integrin beta1 signaling. Biochem Biophys Res Commun. 2012; 426: 461-7.

117. Sakamoto K, Goransson O, Hardie DG, et al. Activity of LKB1 and AMPK-related kinases in skeletal muscle: effects of contraction, phenformin, and AICAR. Am J Physiol Endocrinol Metab. 2004; 287: E310-7.

118. Lee $\mathrm{KH}, \mathrm{Hsu} \mathrm{EC}$, Guh JH, et al. Targeting energy metabolic and oncogenic signaling pathways in triple-negative breast cancer by a novel adenosine monophosphate-activated protein kinase (AMPK) activator. J Biol Chem. 2011; 286: 39247-58

119. Dowling RJ, Zakikhani M, Fantus IG, et al. Metformin inhibits mammalian target of rapamycin-dependent translation initiation in breast cancer cells. Cancer research. 2007; 67: 10804-12.

120. Qu C, Zhang W, Zheng G, et al. Metformin reverses multidrug resistance and epithelial-mesenchymal transition (EMT) via activating AMP-activated protein kinase (AMPK) in human breast cancer cells. Mol Cell Biochem. 2014; 386: 63-71.

121. Rocha GZ, Dias MM, Ropelle ER, et al. Metformin amplifies chemotherapy-induced AMPK activation and antitumoral growth. Clin Cancer Res. 2011; 17: 3993-4005.

122. Adamovich $Y$, Adler J, Meltser V, et al. AMPK couples p73 with p53 in cell fate decision. Cell Death Differ. 2014; 21: 1451-9.

123. Lee YG, Lee SW, Sin HS, et al. Kinase activity-independent suppression of p73alpha by AMP-activated kinase alpha (AMPKalpha). Oncogene. 2009; 28: 1040-52.

124. Yen CS, Chen JC, Chang YF, et al. Lovastatin causes FaDu hypopharyngeal carcinoma cell death via AMPK-p63-survivin signaling cascade. Sci Rep. 2016; 6: 25082.

125. Cerezo M, Tichet M, Abbe P, et al. Metformin Blocks Melanoma Invasion and Metastasis Development in AMPK/p53-Dependent Manner. Molecular cancer therapeutics. 2013; 12: 1605-15.

126. Juan J Gu QZ, Cory Mavis, Myron S. Czuczman and Francisco J. Hernandez-Ilizaliturri. Metformin Induces p53-Dependent Mitochondrial 
Stress in Therapy-Sensitive and -Resistant Lymphoma Pre-Clinical Model and Primary Patients Sample with B-Cell Non-Hodgkin Lymphoma (NHL). Blood. $2015 ; 126$.

127. Ben Sahra I, Laurent K, Giuliano S, et al. Targeting cancer cell metabolism: the combination of metformin and 2-deoxyglucose induces p53-dependent apoptosis in prostate cancer cells. Cancer research. 2010; 70: 2465-75.

128. Buzzai M, Jones RG, Amaravadi RK, et al. Systemic treatment with the antidiabetic drug metformin selectively impairs p53-deficient tumor cell growth. Cancer research. 2007; 67: 6745-52.

129. Jeong YK, Kim MS, Lee JY, et al. Metformin Radiosensitizes p53-Deficient Colorectal Cancer Cells through Induction of G2/M Arrest and Inhibition of DNA Repair Proteins. PloS one. 2015; 10: e0143596.

130. Gorrini C, Harris IS, Mak TW. Modulation of oxidative stress as an anticancer strategy. Nat Rev Drug Discov. 2013; 12: 931-47.

131. Algire C, Moiseeva O, Deschenes-Simard X, et al. Metformin reduces endogenous reactive oxygen species and associated DNA damage. Cancer Prev Res (Phila). 2012; 5: 536-43.

132. Piwkowska A, Rogacka D, Jankowski M, et al. Metformin induces suppression of $\mathrm{NAD}(\mathrm{P}) \mathrm{H}$ oxidase activity in podocytes. Biochem Biophys Res Commun. 2010; 393: 268-73.

133. Hou X, Song J, Li XN, et al. Metformin reduces intracellular reactive oxygen species levels by upregulating expression of the antioxidant thioredoxin via the AMPK-FOXO3 pathway. Biochem Biophys Res Commun. 2010; 396: 199-205.

134. Candi E, Rufini A, Terrinoni A, et al. Differential roles of p63 isoforms in epidermal development: selective genetic complementation in p63 null mice. Cell death and differentiation. 2006; 13: 1037-47.

135. Wang X, Mori I, Tang W, et al. p63 expression in normal, hyperplastic and malignant breast tissues. Breast Cancer. 2002; 9: 216-9.

136. Koga F, Kawakami S, Fujii Y, et al. Impaired p63 expression associates with poor prognosis and uroplakin III expression in invasive urothelial carcinoma of the bladder. Clin Cancer Res. 2003; 9: 5501-7.

137. Maas AM, Bretz AC, Mack E, et al. Targeting p73 in cancer. Cancer Lett. 2013; 332: 229-36.

138. Bergamaschi D, Gasco M, Hiller L, et al. p53 polymorphism influences response in cancer chemotherapy via modulation of p73-dependent apoptosis. Cancer Cell. 2003; 3: 387-402.

139. Rosenbluth JM, Mays DJ, Pino MF, et al. A gene signature-based approach identifies mTOR as a regulator of p73. Mol Cell Biol. 2008; 28: 5951-64.

140. Hanna RK, Zhou C, Malloy KM, et al. Metformin potentiates the effects of paclitaxel in endometrial cancer cells through inhibition of cell proliferation and modulation of the mTOR pathway. Gynecologic oncology. 2012; 125: 458-69. 